$03,04,11,16$

\title{
О возможных состояниях кристаллической структуры, предшествующих фазовому переходу в кристаллах $\mathrm{Zn}_{1-x} \mathrm{~V}_{x} \mathrm{Se}$ $(0.01 \leq x \leq 0.10)$
}

\author{
(C) В.И. Максимов, Е.Н. Максимова, Т.П. Суркова, А.П. Вохмянин
}

Институт физики металлов им. М.Н. Михеева УрО РАН,

Екатеринбург, Россия

E-mail: kokailo@rambler.ru

(Поступила в Редакцию 20 июня 2018 г.)

В результате подробного нейтронографического исследования объемных полупроводниковых кристаллов $\mathrm{ZnSe}$ с повышенным содержанием ванадия, охарактеризованы проявившиеся систематические новые образования в обратной решетке кубической структурной модификации соединения II-VI. Впервые получены прямые доказательства того, что обнаруживаемые нейтронным рассеянием на исследованных кристаллах дополнительные узлы $k=(1 / 31 / 31 / 3) 2 \pi / a(k-$ волновой вектор, $a$ - параметр кубической элементарной ячейки) в случае их принадлежности к взаимопроникающим повернутым подрешеткам содержат сверхструктурный вклад, формируемый коротковолновыми деформациями. Определяя описанное структурное состояние кристаллов как предпереходное к концентрационной фазовой трансформации ГЦК ГПУ, и рассматривая переходы по звезде волнового вектора $k_{5}$ ГЦК-решетки, для перехода по однолучевому каналу указаны базисные функции, позволяющие анализировать атомные смещения, корреляции между которыми порождают сверхструктуры дисторсионного типа.

Работа выполнена с использованием УНУ „НМК ИФМ“ в рамках государственного задания по темам „Поток“ Г.р. АААА-А18-118020190112-8 и „Электрон“ Г.р. № АААА-А18-118020190098-5.

DOI: 10.21883/FTT.2019.01.46892.169

\section{1. Введение}

Легированные $\mathrm{Zn}_{1-x} M e_{x}^{3 d} B^{\mathrm{VI}}\left(M e^{3 d}=\mathrm{V}, \mathrm{Cr}, \mathrm{Mn}, \mathrm{Fe}\right.$ и др., $B^{\mathrm{VI}}=\mathrm{O}, \mathrm{S}$, $\mathrm{Se}, \mathrm{Te})$ принято относить к разбавленным магнитным полупроводникам (РМП), известным своими уникальными электронными и магнитооптическими свойствами, широко используемыми на практике [1-3]. Попытки применить перечисленные выше РМП для создания элементов полупроводниковой спинтроники [4,5] сместили сегодня акцент на исследование объектов с пониженной размерностью, появление свидетельств о высокотемпературном ферромагнетизме которых в последнее время участилось в печати [6-10]. Поэтому детальное изучение свойств легированных $3 d$-ионами соединений II-VI остается актуальным.

Две модификации халькогенидов цинка - кубического сфалерита (цинковой обманки) и гексагонального вюрцита - представляют их полиморфизм [11]. Решетка в одном случае гранецентрированная кубическая (ГЦК), в другом - строящаяся по принципу гексагональной плотнейшей упаковки (ГПУ). Обе структуры относятся к разным категориям симметрии, но практически не различимы в локальной тетраэдрической координации атомов $[11,12]$. Для исходных соединений цинка с серой и селеном при нормальных условиях более стабильна кубическая фаза $[12,13]$.

Магнитные ионы с недостроенными $d$-, $f$-электронными оболочками в легированных халькогенидах цинка обычно замещают в исходных структурах катионы, на что указывают результаты оптических исследований [14]. При слабом легировании объемных полупроводников $\mathrm{Zn} B^{\mathrm{VI}}$ элементами $3 d$-ряда (тысячные или даже десятитысячные доли в формульной единице), можно считать, что структурную модификацию в равновесном состоянии определяет матрица легирования. Однако, свойственная матрицам $\mathrm{Zn} B^{\mathrm{VI}}\left(B^{\mathrm{VI}}=\mathrm{S}, \mathrm{Se}, \mathrm{Te}\right)$,капризность синтеза“ создает сложность в понимании результатов формирования физических свойств при их разбавлении магнитоактивной примесью. Реальная структура этих кристаллов допускает локальные нарушения последовательности укладки плотноупакованных слоев, одновременное присутствие кристаллитов кубической и гексагональной модификации, неоднофазность вследствие политипии [14-16]. Основная причина, препятствующая получению „идеальной“ кристаллической фазы рассматриваемых полупроводников, кроется, по-видимому, в легкости, с которой формируются нарушения в послойной укладке атомов. В этом отношении наиболее изученный для них полиморфизм является хорошей иллюстрацией. Оценки энергии стабилизации кубической и гексагональной модификаций для каждого конкретного соединения в ряду $\mathrm{Zn} B^{\mathrm{VI}}$ дают весьма близкие значения [13]. Информация о собственных дефектах в „чистых“ матрицах II-VI довольно подробно представлена в $[16,17]$.

Точные фазовые диаграммы наиболее изученных систем $\mathrm{Zn}_{1-x} \mathrm{Mn}_{x} B^{\mathrm{VI}}\left(B^{\mathrm{VI}}=\mathrm{S}, \mathrm{Se}\right)$ по мере повышения содержания марганца, обладающего из $3 d$-металлов наилучшей растворимостью в соединениях II-VI, показыва- 
ют область гексагональной фазы со структурой вюрцита (в случае $\mathrm{ZnSe}$ кубическая модификация сохраняется до концентраций $\mathrm{Mn} x \approx 0.35$, а для $\mathrm{ZnS}-0.10$ в формульной единице) [12]. Указанный факт позволяет говорить о концентрационном фазовом переходе ГЦК-ГПУ в этих системах. С симметрийной точки зрения названный переход относится к реконструктивным. В рассматриваемых системах его результатом является изменение типа укладки координационных тетраэдров [18].

Магнитоактивные $3 d$-ионы, $d$-электронная оболочка которых, в отличие от $\mathrm{Mn}^{2+}$, не обладает сферической симметрией, гораздо хуже растворяются в объемных кристаллах соединений $\mathrm{Zn} B^{\mathrm{VI}}$. Практически все монохалькогениды $3 d$-металлов, если они существуют, не кристаллизуются в структуры халькогенидов цинка. Для атомного порядка соединений $M e B^{\mathrm{VI}}(M e-3 d$-металл) наиболее характерен структурный тип NiAs [18-20]. Taким образом, проблема низкой растворимости $3 d$-ионов $\mathrm{V}, \mathrm{Cr}, \mathrm{Fe}$ и т.д. в кубических кристаллах полупроводников II-VI связана с известной проблемой изодиморфизма [18]: чтобы растворить примесь в матрице легирования, необходимо трансформировать ее халькогенид в структуру сфалерита (т. е. совершить полиморфное превращение, затратив на него некоторую энергию), а лишь затем позволить ему заместить основной компонент в кристалле, на что опять же потребуется дополнительное поглощение энергии, связанное с „геометрическими“ и „электронными“ видами деформации в конечном продукте. Именно поэтому экспериментальные попытки получить высокие уровни легирования $3 d$-ионов в матрице II-VI приводят к образованию преципитатов соответствующих халькогенидов $3 d$-элементов [21].

Ранее, на монокристаллах $\mathrm{Zn}_{1-x} M e_{x} B^{\mathrm{VI}}(M e=\mathrm{V}$, $\mathrm{Cr}, \mathrm{Fe}, \quad$ и т.д. кроме $\mathrm{Mn} ; B^{\mathrm{VI}}=\mathrm{O}, \quad \mathrm{Se}, \mathrm{Te}$; $0.0002 \leq x \leq 0.005)$, нами было показано, что в интервале температур $100-300 \mathrm{~K}$ диффузное рассеяние тепловых нейтронов в окрестности сильных брэгговских рефлексов является главным признаком сильной дестабилизации исходной кристаллической структуры и указывает на сформированные в ней наноразмерные области неоднородных искажений, геометрия которых зависит от $3 d^{n}$-конфигурации ионов примеси [22,23]. С увеличением концентрации примеси число поврежденных нанообластей в структуре увеличивается, а среди атомных смещений усиливается беспорядок [24-29]. Обнаруженные в обратной решетке кубических кристаллов $\mathrm{Zn}_{1-x} M e_{x} B^{\mathrm{VI}}$ систематические дополнительные узлы с волновыми векторами $k=(1 / 31 / 31 / 3) 2 \pi / a \quad(a-$ параметр элементарной ячейки) позволяют интерпретировать структурное состояние как предшествуюшее концентрационному фазовому переходу ГЦК-ГПУ [2630]. Названная трансформация наблюдается в системах $M e_{1-x} \mathrm{Zn}_{x} \mathrm{O}$ при постепенном замещении $3 d$-металла немагнитным цинком в оксиде с исходной структурой каменной соли [31,32]. Поэтому в настоящей работе нами была поставлена задача с помощью нейтронов более детально охарактеризовать структурное состояние, предшествующее возможному фазовому превращению в кристаллах $\mathrm{Zn}_{1-x} \mathrm{~V}_{x} \mathrm{Se}(0.01 \leq x \leq 0.10)$ с относительно высоким содержанием легирующей добавки. Решение данной задачи представляет особый фундаментальный интерес, поскольку касается уникального случая изодиморфизма, когда исходный чистый компонент, в котором ведется замещение, имеет две полиморфные модификации с кристаллическими структурами разных симметрий, а значения энергий стабилизации обоих модификаций слабо отличаются друг от друга. При этом для обоих „крайних“ (чистых) составов изодиморфического ряда общим является построение анионной подрешетки по принципу плотнейшей упаковки.

\section{2. Образцы и методика эксперимента}

Использованные в работе кристаллы $\mathrm{Zn}_{1-x} \mathrm{~V}_{x} \mathrm{Se}$ представляли собой образцы неправильной формы с линейными размерами $\sim 0.5 \mathrm{~cm}$ в трех взаимно перпендикулярных направлениях, выращенные методом химического транспорта из газовой фазы.

Эксперименты по упругому рассеянию тепловых нейтронов были выполнены на специальном многоканальном дифрактометре для исследований монокристаллов. Длина волны падающего на образец пучка нейтронов, сформированного двойным кристалломмонохроматором из пиролитического графита и деформированного германия, составляла $1.57 \AA \AA$. Эффективная монохроматизация первичного пучка и оптимальный выбор длины волны нейтронов позволили подавить на дифракционной картине кристалла эффекты кратных дифракционных гармоник, что позволило существенно повысить чувствительность нашей нейтронографической методики.

\section{3. Результаты нейтронного эксперимента и их обсуждение}

Нейтронографическая аттестация кристаллов $\mathrm{Zn}_{1-x} \mathrm{~V}_{x} \mathrm{Se}(x=0.01 ; 0.05 ; 0.10)$ показала, что основной структурный мотив во всех трех случаях следует считать кубическим. Из измерений кривых отражения интенсивных брэгговских рефлексов, при наличии объемного фрагмента „совершенного“ кристалла в каждом образце, для кристаллов с $x=0.05$ и $x=0.10$ сильно проявляется микроблочность. Плоские грани (сколы) при содержании ванадия $x=0.01$ с наибольшей вероятностью отвечали плоскостям $\{100\}$, при содержании $x=0.05-\{100\}$ и $\{111\}$, при $x=0.10-\{111\}$.

Основные картины нейтронной дифракции были измерены в обратной решетке кристаллов $\mathrm{Zn}_{1-x} \mathrm{~V}_{x} \mathrm{Se}$ $(x=0.01 ; 0.05 ; 0.10)$ на плоскости $(0 \overline{1} 1)$. На рис. 1 приведены картины рассеяния при комнатной температуре между основными структурными узлами обратной решетки вдоль направлений $\langle 111\rangle$. На представленных картинах вертикальные шкалы нормированы по пиковой интенсивности самого интенсивного структурного 


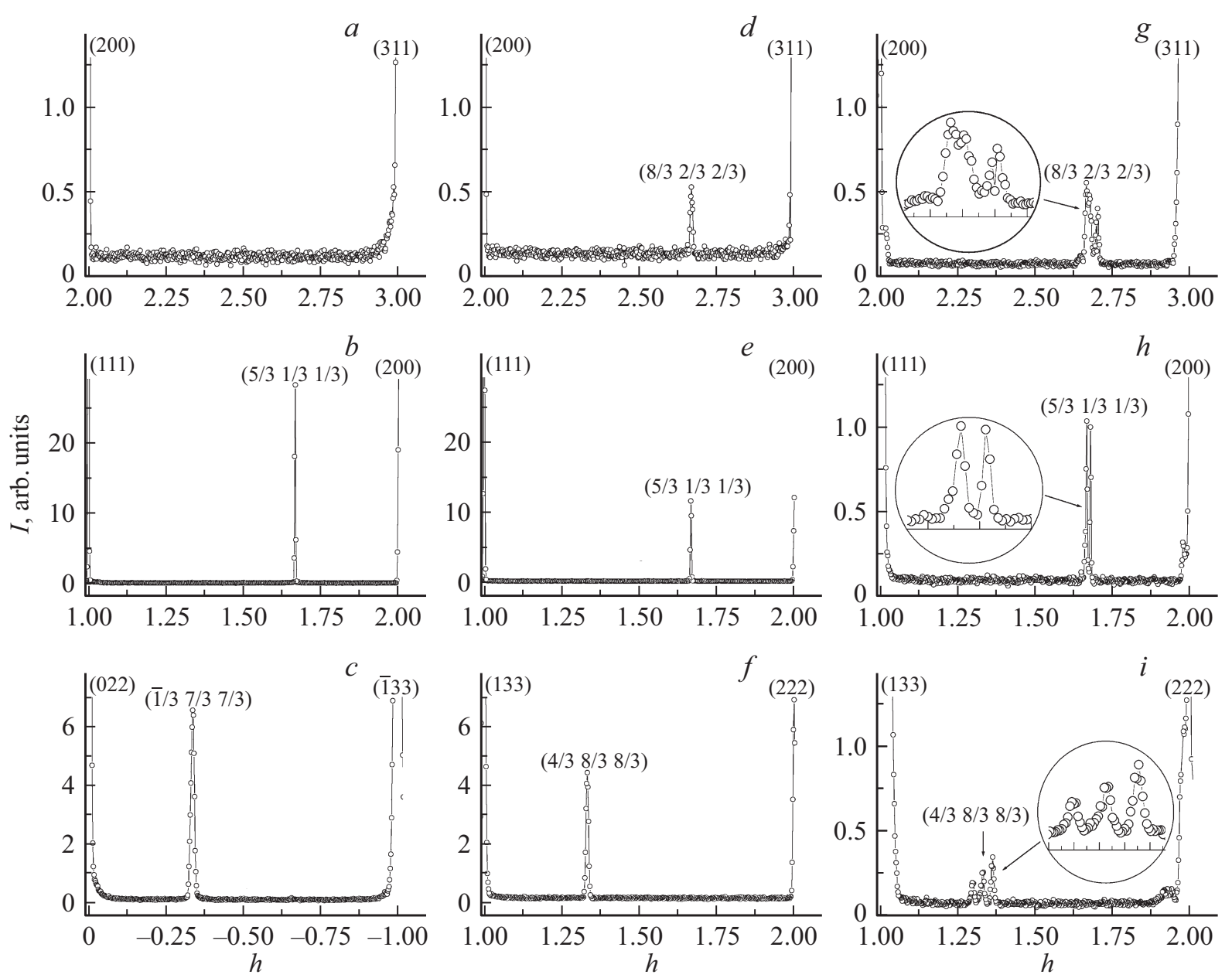

Рис. 1. Картины нейтронной дифракции монокристаллов $\mathrm{Zn}_{1-x} \mathrm{~V}_{x} \mathrm{Se}(a, b, c-x=0.01 ; d, e, f-x=0.05 ; g, h, i-x=0.10)$ в сечении $(0 \overline{1} 1)$ обратной решетки при $300 \mathrm{~K}$, измеренные между узлами $(200)-(311)(a, d, g),(111)-(200)(b, e, h),(022)-(\overline{1} 33)(c)$, $(133)-(222)(f, i)$ вдоль направлений $\langle 111\rangle$. На вставках $g-i$ приведены профили дополнительных отражений в увеличенном масштабе.

рефлекса (111), которая принята равной 100. Обнаруживаемые дополнительные отражения соответствуют волновым векторам

$$
k=(1 / 31 / 31 / 3) 2 \pi / a,
$$

где $a$ - параметр кубической элементарной ячейки. Количество и интенсивность этих отражений, а также расположение на плоскости, при разном содержании ванадия разное. На дифрактограмме вдоль прямой между узлами (200) и (311) кристалла с содержанием ванадия $x=0.01$ нет никаких дополнительных эффектов рассеяния (рис. $1, a$ ), в то время как для кристалла с $x=0.05$ на той же траектории (рис. $1, d$ ) появляется рефлекс в сверхструктурном положении (8/32/32/3). Как видно из рис. $1, b$, для кристалла $x=0.01$ пиковая интенсивность дополнительного отражения, соответствующего сверхструктурному узлу $(5 / 31 / 31 / 3)$, достигает порядка $30 \%$ от максимума самого интенсивного структурного рефлекса (111). В случае кристалла с $x=0.05$ максимум интенсивности этого же дополнительного отражения относительно интенсивности рефлекса (111) составляет $10 \%$ (рис. $1, e$ ).

Ранее, в работах [25-30], во всех случаях мы трактовали представленные на рис. 1 дополнительные эффекты рассеяния как сверхструктурные рефлексы и рассматривали их как свидетельство зарождения в кристаллах предпереходного состояния к структурной концентрационной фазовой трансформации ГЦК-ГПУ. Однако, наблюдаемые весьма высокие интенсивности сверхструктурных отражений от кристаллов $\mathrm{Zn}_{1-x} \mathrm{~V}_{x} \mathrm{Se}$ $(x=0.01 ; 0.05)$, при отсутствии рефлексов гексагональной фазы, требуют уточнения нашего первоначального понимания структурного состояния рассматриваемых полупроводниковых кристаллов с повышенным уровнем легирования.

На рис. 2 приведены полученные в рамках нашего нейтрон-дифракционного эксперимента схемы расположения дополнительных узлов, определяемых векторами (1) в обратной решетке сечением плоскостью (011) для всех трех исследованных кристаллов $\mathrm{Zn}_{1-x} \mathrm{~V}_{x} \mathrm{Se}$ $(x=0.01 ; 0.05 ; 0.10)$. 

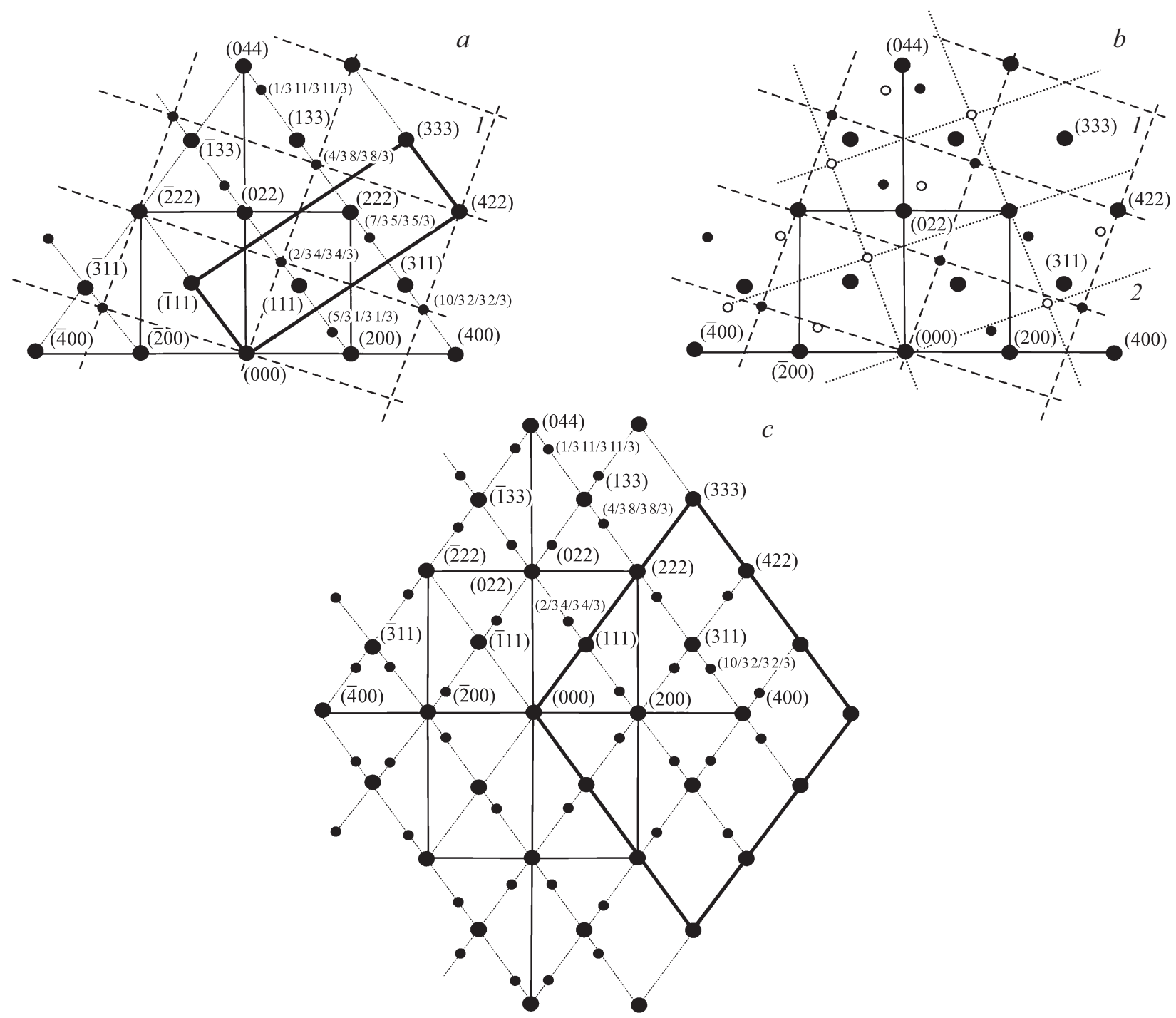

Рис. 2. Картины обратной решетки ГЦК монокристаллов $\mathrm{Zn}_{1-x} \mathrm{~V}_{x} \mathrm{Se}(a-x=0.01 ; b-x=0.05 ; c-x=0.10)$ в сечении плоскостью $(0 \overline{1} 1)$, уточненные в результате нейтронографического эксперимента. Крупные темные кружки - основные узлы. Темные кружки меньшего размера - сверхструктурные положения, соответствующие дополнительным отражениям, отвечающим систематике $k=(1 / 31 / 31 / 3) 2 \pi / a$. Проявленные проникающие подрешетки показаны штриховой линией $(1-a)$ и штриховой и пунктирной линиями $(1$ и $2-b)$ соответственно. При содержании ванадия $x=0.05(b)$ дополнительные узлы, формирующие подрешетку 2, обозначены светлыми кружками. Более тонкие эффекты (расщепления, узлы длинноволновых модулированных структур) для кристалла с $x=0.10(c)$ опущены. Жирными линиями на схемах $a$ и $c$ показаны результирующие элементарные ячейки.

Из схемы рис. 2, $a$ нетрудно обнаружить, что попарно взятые радиус-векторы, определяющие положения сверхструктурных и структурных узлов $(5 / 31 / 31 / 3)$ и (111), $(2 / 34 / 34 / 3)$ и $(200),(10 / 32 / 32 / 3)$ и $(222)$, и т.д. равны. При обсуждении рассеяния нейтронов на кристаллах $\mathrm{Cd}_{1-x} \mathrm{Mn}_{x} \mathrm{Te}$ в [12] подобные дополнительные узлы с векторами (1) вполне резонно интерпретировались как результат от двойниковых включений, плоскостями двойникования в которых являются плоскости плотной упаковки $\{111\}$, а направлениями сдвига $-\langle\overline{2} 11\rangle$. Появление дополнительных дифракционных отражений вследствие указанного двойникования наглядно иллюстрирует схема рис. 3 . Известно, что генерация двойника в структуре сфалерита при сдвиге на плоскости (111) вдоль направления $[\overline{2} 11]$ происходит как поворот структуры двойникового фрагмента на $180^{\circ}$ относительно исходной решетки. Описывая структуру сфалерита через общепринятые обозначения укладки атомных слоев в ГЦК-решетку ...ABC ... (где $A, B, C$ - двухатомные слои халькогена и металла с атомами разного сорта в точности один под другим, сложенные по принципу плотнейшей упаковки), формирование двойника передает запись ...ABC/BACBA .., т. е. обращение последовательности укладки $[19,33]$. Поэтому в ОЦК-решетке, являющейся обратной к ГЦК, поворот какого-либо фрагмента на $180^{\circ}$ приведет, согласно схеме 3 , к появлению 


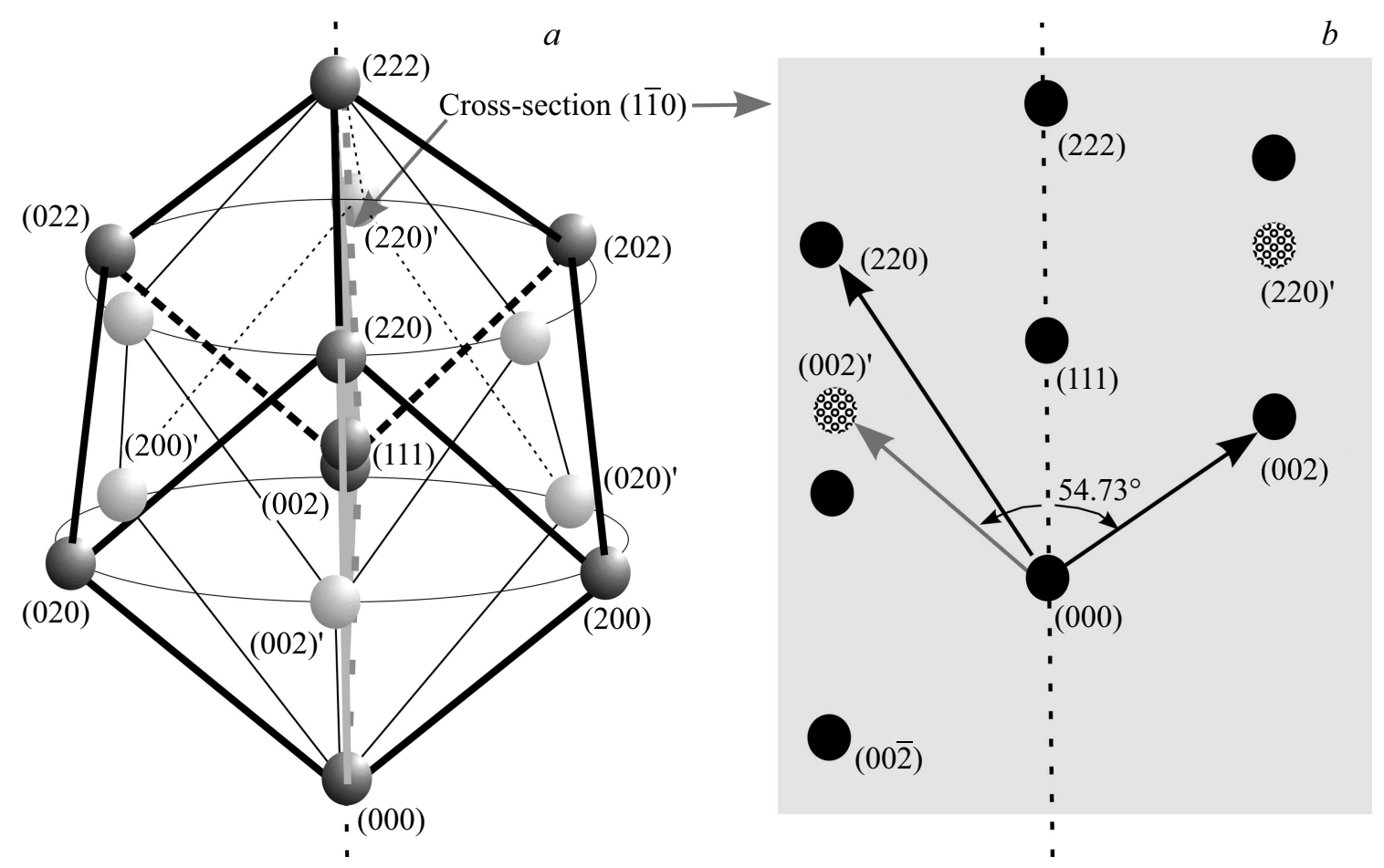

Рис. 3. Схема, поясняющая появление дополнительных узлов в обратной решетке, отвечающей кубической структуре сфалерита в случае двойникования: $a-$ поворот ячейки Бравэ вокруг оси [111] на $180^{\circ}$ для решетки, обратной к ГЦК (основная и повернутая ячейки проведены жирными и тонкими линиями соответственно); $b$ - проекция решетки, обратной к ГЦК, в сечении плоскостью (110) в результате поворота ее фрагмента на $180^{\circ} \mathrm{C}$ (темные кружки - узлы основной решетки, заштрихованные - узлы повернутого фрагмента).

„дополнительных“ узлов в сечениях плоскостями $\{110\}$. Из схемы $3 b$ легко видеть, что в обратном пространстве эти узлы соответствуют волновым векторам (1) и, таким образом, на дифракционной картине ГЦКкристалла должны появиться отвечающие им отражения. На плоскостях $\{110\}$ эти отражения составляют „Подрешетки“, повернутые относительно основной решетки на „кубический“ угол между плоскостями $\{111\}$, равный $109.47^{\circ}$. Описание дифракции от такого двойникования приведено в [12]. При этом все решетки - основная и „повернутые“ - являются взаимопроникающими по отношению друг к другу.

Анализ политипии соединений II-VI [33,34 и ссылки в этих работах] показывает, что основной чертой дифракции является перераспределение интенсивности структурных пиков вследствие увеличения периода вдоль „гексагональной“ оси $c$. Таким образом, наблюдение дополнительных монокристалльных отражений, соответствующих на рис. 2 узлам (1) в обратном пространстве, может указывать также и на формирование политипных последовательностей укладки атомных слоев с периодом кратным 3. Следовательно, узлы системы (1), образующие повернутые взаимопроникающие решетки в плоскости (011) кристаллов $\mathrm{ZnSe}$ с повышенным уровнем легирования магнитоактивными $3 d$-ионами, не являются „чисто“ сверхструктурными, понимая „сверхструктурную“ природу исключительно как следствие коррелированных атомных смещений.
Для кристалла $\mathrm{Zn}_{1-x} \mathrm{~V}_{x} \mathrm{Se}(x=0.01)$ система узлов (1) в плоскости $(0 \overline{1} 1)$, представляет собой „подрешетку“ (проведенную пунктиром и обозначенную 1 на рис. $2, a)$, повернутую относительно основной решетки именно так, как если бы ее происхождение обуславливалось выше описанным двойникованием. В выбранной системе отсчета поворот структуры на $180^{\circ}$ происходит вокруг оси $[\overline{1} 11]$, соответственно, сдвиг осуществляется на плоскости (111). В обратной решетке кристалла с содержанием ванадия $x=0.05$, на плоскости (011) проявляется „вторая“ повернутая подрешетка (обозначенная 2 на рис. $2, b)$. В модели двойникования ей соответствует сдвиг на плоскости (111). Рефлексы, представляющие подрешетку 2, на порядок слабее рефлексов, определяющих узлы подрешетки 1. При самом высоком содержании ванадия $(x=0.10)$ дополнительные узлы $g-i$, принадлежащие систематике (1), как видно из данных рис. 1, имеют собственную тонкую структуру. Эта тонкая структура, для представленных конкретных случаев (вставки на рис. $1, h, i)$, показывает два вида расщепления: либо как собственно расщепление на два „подузла“, либо как появление сателлитов. (Детали расщепления более подробно обсуждаются в работах [26,30].) При этом для обоих подрешеток сравнение интенсивностей рефлексов, идентичных в описании двойникования, с учетом расщепления показывает их соизмеримость по порядку величины. 

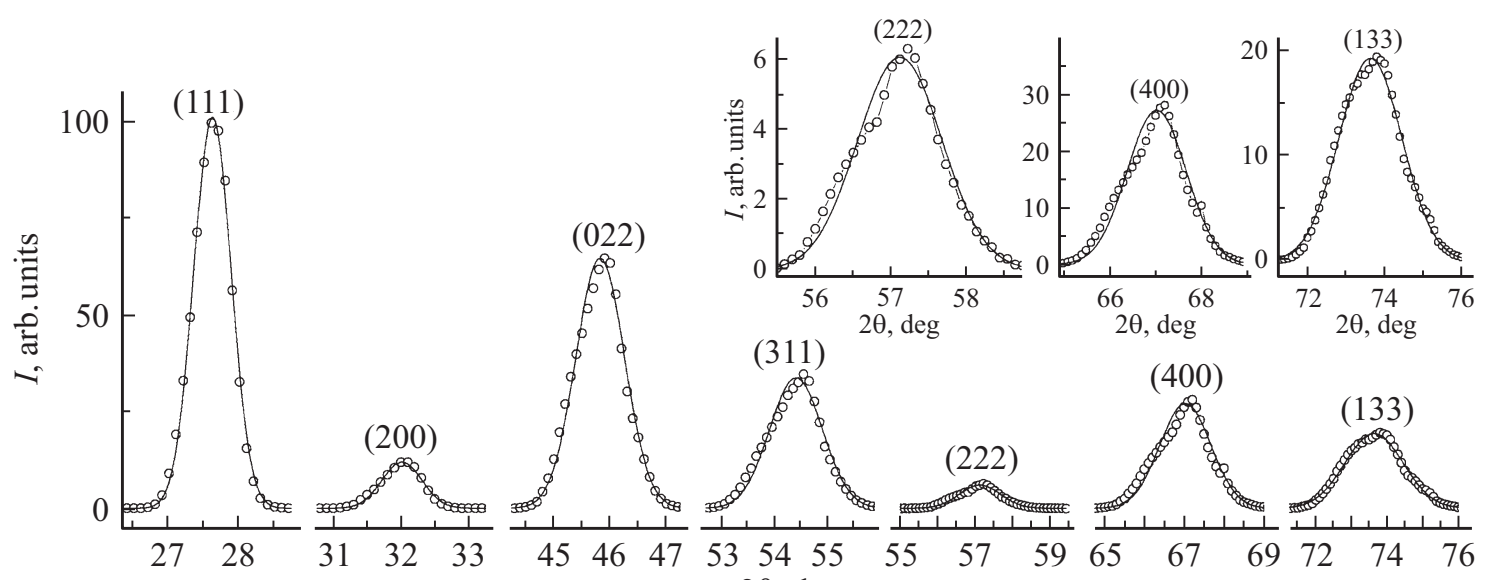

(311)
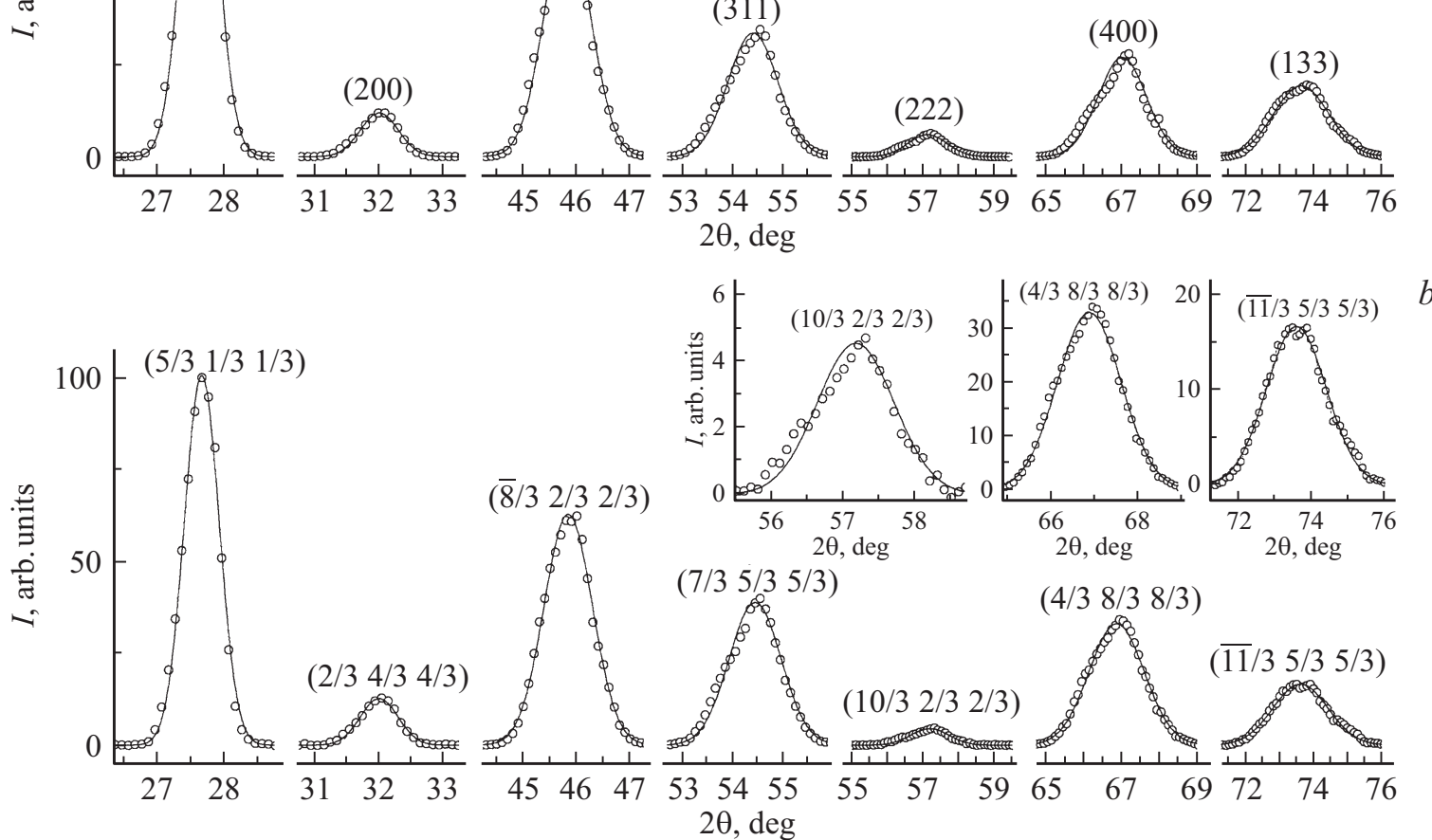

$(4 / 38 / 38 / 3)$

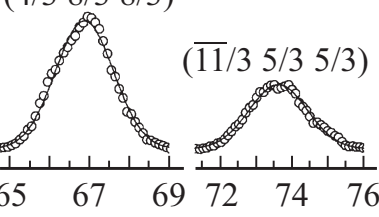

Рис. 4. Картины нейтронной дифракции монокристалла $\mathrm{Zn}_{0.95} \mathrm{~V}_{0.05} \mathrm{Se}$, измеренные в плоскости (01̄1) при $300 \mathrm{~K}$ относительно основных $(a)$ и дополнительных $(b)$, относящихся к систематике $k=(1 / 31 / 31 / 3) 2 \pi / a$ узлов обратной решетки в радиальных направлениях. Вставки иллюстрируют профили соответствующих отражений в увеличенном масштабе.

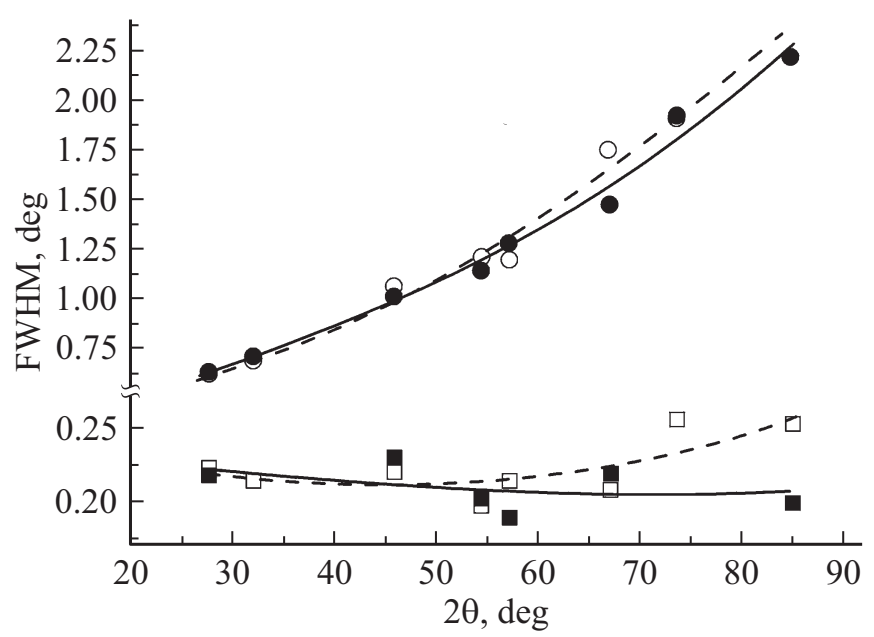

Рис. 5. Полуширины профилей рефлексов, отвечающих узлам основной решетки (темные символы) и проникающей подрешетки 1 (светлые символы), измеренные в плоскости $(0 \overline{1} 1)$ в радиальном (кружки) и тангенциальном (квадраты) направлениях.
Чтобы прояснить природу наблюдаемых дополнительных отражений, на кристалле $\mathrm{Zn}_{0.95} \mathrm{~V}_{0.05} \mathrm{Se}$ мы провели подробную нейтронографическую съемку структурных рефлексов и ярких дополнительных отражений, определяющих повернутую решетку 1 . На рис. 4 приведены результаты сканирования структурных рефлексов и дополнительных отражений (задающих подрешетку 1) кристалла $\mathrm{Zn}_{0.95} \mathrm{~V}_{0.05} \mathrm{Se}$, измеренные вдоль радиальных направлений при нормальных условиях. Как видно из полученных данных, соотношение пиковых интенсивностей дополнительных отражений качественно повторяет дифракционную картину от основной решетки. Значения полуширин рефлексов, измеренных в тангенциальных и радиальных направлениях (рис. 5), для обеих решеток совпадают по порядку величины и на качественном уровне следуют соответствующим функциям разрешения дифрактометрической установки. Полученный результат наглядно показывает, что происхождение повернутой решетки 1 кристалла $\mathrm{Zn}_{0.95} \mathrm{~V}_{0.05} \mathrm{Se}$ в основном обуславливает двойникование.

Деформированное состояние на профилях рефлексов, определяющих подрешетку 1, выражено слабее, чем на профилях основных структурных рефлексов. Детальное 
Таблица 1. Характеристики основных и дополнительных отражений кристалла $\mathrm{Zn}_{0.95} \mathrm{~V}_{0.05} \mathrm{Se}$, измеренных в плоскости (01̄1) при $T=300 \mathrm{~K}$

\begin{tabular}{|c|c|c|c|c|c|c|c|}
\hline \multicolumn{8}{|c|}{ Основная решетка } \\
\hline $\begin{array}{l}\quad \text { Рефлексы } \\
I_{\mathrm{m}}, \text { arb. units } \\
I_{\mathrm{m}}, \text { norm } \\
I_{\mathrm{I}} \text {, arb. units } \\
I_{\mathrm{I}}, \text { norm }\end{array}$ & $\begin{array}{c}111 \\
75400 \\
100 \\
50000 \\
100\end{array}$ & $\begin{array}{c}200 \\
9000 \\
12 \\
6500 \\
13\end{array}$ & $\begin{array}{c}022 \\
48200 \\
64 \\
50500 \\
101\end{array}$ & $\begin{array}{c}311 \\
26450 \\
35 \\
31500 \\
63\end{array}$ & $\begin{array}{l}222 \\
4500 \\
6 \\
6200 \\
12\end{array}$ & $\begin{array}{c}400 \\
21100 \\
28 \\
31500 \\
63\end{array}$ & $\begin{array}{c}133 \\
14350 \\
19 \\
28500 \\
57\end{array}$ \\
\hline \multicolumn{8}{|c|}{ Подрешетка 1} \\
\hline $\begin{array}{l}\quad \text { Рефлексы } \\
I_{\mathrm{m}}, \text { arb. units } \\
I_{\mathrm{m}}, \text { norm } \\
I_{\mathrm{I}} \text {, arb. units } \\
I_{\mathrm{I}}, \text { norm }\end{array}$ & $\begin{array}{c}5 / 31 / 31 / 3 \\
9500 \\
100 \\
6100 \\
100\end{array}$ & $\begin{array}{c}2 / 34 / 34 / 3 \\
1240 \\
13 \\
900 \\
15\end{array}$ & $\begin{array}{c}\overline{8} / 32 / 32 / 3 \\
5900 \\
62 \\
6500 \\
107\end{array}$ & $\begin{array}{c}7 / 35 / 35 / 3 \\
3800 \\
40 \\
4600 \\
75\end{array}$ & $\begin{array}{c}10 / 32 / 32 / 3 \\
450 \\
4.5 \\
550 \\
9\end{array}$ & $\begin{array}{c}4 / 38 / 38 / 3 \\
3200 \\
33 \\
5500 \\
90\end{array}$ & $\begin{array}{c}11 / 35 / 35 / 3 \\
1550 \\
16 \\
3100 \\
51\end{array}$ \\
\hline \multicolumn{8}{|c|}{ Соотношения характеристик } \\
\hline $\begin{array}{l}I_{\mathrm{m}, \text { sublatt }} / I_{\mathrm{m}, \text { struct }}, \% \\
\left(I_{\mathrm{m}, \text { struct }}-I_{\mathrm{m}, \text { sublat }}\right)_{\text {norm }} \\
I_{\mathrm{I}, \text { sublatt }} / I_{\mathrm{I}, \text { struct }} \% \\
\left(I_{\mathrm{I}, \text { struct }}-I_{\mathrm{I}, \text { sublatt }}\right)_{\text {norm }}\end{array}$ & $\begin{array}{c}12 \\
0 \\
12 \\
0\end{array}$ & $\begin{array}{l}14 \\
-1 \\
14 \\
-2\end{array}$ & $\begin{array}{c}12 \\
2 \\
13 \\
-6\end{array}$ & $\begin{array}{c}14 \\
-5 \\
15 \\
-12\end{array}$ & $\begin{array}{c}10 \\
1.5 \\
9 \\
3\end{array}$ & $\begin{array}{l}15 \\
-5 \\
17 \\
-27\end{array}$ & $\begin{array}{c}11 \\
3 \\
11 \\
6\end{array}$ \\
\hline
\end{tabular}

описание профилей структурных брэгговских отражений, измеренных в радиальных направлениях, для трех исследованных в данной работе кристаллов $\mathrm{Zn}_{1-x} \mathrm{~V}_{x} \mathrm{Se}$ $(x=0.01 ; 0.05 ; 0.10)$ содержится в [29]. Однако, наибольшие различия рефлексов основной и „повернутой“ решеток проявились в соотношениях интегральной интенсивности.

Интенсивности основных и дополнительных отражений кристалла $\mathrm{Zn}_{0.95} \mathrm{~V}_{0.05} \mathrm{Se}$ при $T=300 \mathrm{~K}$ характеризуют данные табл. 1. Значения максимальной интенсивности двух наборов рефлексов взяты из данных измерений в радиальных направлениях, сканированных в статистически одинаковых условиях. Поскольку характеристики на рис. 5 для двух решеток практически совпадают, значения интегральной интенсивности рассчитывались по этим же данным. Для оценки интегральной интенсивности использовались инструменты программы Winplotr версии 2011 [35], универсальные для работы с дифрактограммами.

Как видно из табл. 1, максимумы интенсивности подрешетки 1, в сопоставлении пиковым значениям рефлексов основной решетки в соответствие с описанием двойникования, составляют около $10 \%$ от структурных отражений (строка 11 таблицы). При этом из сравнения данных от двух решеток следует, что разница максимальных нормированных значений не превышает 5\% (строка 12 таблицы). Сопоставление ,абсолютных“ значений интегральной интенсивности для двух решеток, казалось бы, вносит только незначительную нерегулярность в набор расхождений (строка 13 таблицы). Однако же, характеристики сравнения наборов нормированных ее значений оказываются более чувствительными к различиям в интенсивности рефлексов, показывая максимум в 27\% (строка 14 таблицы). Такие явные различия характеристик сравнения, при существенном разбросе в составляющем их наборе, подразумевают, что причина появления подрешетки 1 кроется не только в двойниковании. Проявления политипии, упомянутой выше, в чистом виде на картинах нейтронной дифракции рассматриваемого кристалла, если бы они были, должны были бы, во-первых, проявиться в отражениях другой систематики [30] и, во-вторых, сильно сказаться на характеристиках сравнения пиковых интенсивностей [34]. Поэтому, наиболее вероятным вкладом в рефлексы повернутой решетки 1, учитывая повышенный уровень легирования кристалла ванадием, является вклад со стороны коррелированных атомных смещений. Таким образом, выявленные заметные различия в поведении интенсивности наборов основных и дополнительных рефлексов через их интегральные характеристики прямо указывают на сверхструктурный вклад в подрешетку 1. Вместе с тем, названный вклад, как следует из данных табл. 1 в целом, достаточно мал, чтобы сказываться непосредственно на значениях максимальной интенсивности в совокупности дополнительных рефлексов, наблюдаемых в сверхструктурных положениях.

Учитывая описанный выше анализ соотношений интенсивностей рефлексов основной и повернутой решеток кристалла $\mathrm{Zn}_{0.95} \mathrm{~V}_{0.05} \mathrm{Se}$, вновь обратимся к данным рис. 2. Напомним, в исследованных кристаллах $\mathrm{ZnSe}$ содержится значительное количество $3 d$-примеси, для моноселенида которой характерна гексагональная структура с „каркасом“ из плотной упаковки атомов халькогена, где катионы предпочитают занимать октаэдрическую позицию. Поэтому, какой бы ни была основная природа наблюдаемых ярких дополнительных отражений, структурное состояние уместно определять как предпереходное (предшествующее фазовой транс- 
Таблица 2. Базисные функции неприводимых представлений группы с $k=(1 / 31 / 31 / 3) 2 \pi / a$ для луча $k_{2}=(0.33-0.33-0.33)$ в овеществленном виде

\begin{tabular}{c|c|c|c|c|c}
\hline \multirow{2}{*}{ Позиция } & \multirow{2}{*}{ Представление } & \multicolumn{4}{|c}{ Атом } \\
\cline { 3 - 6 } & & 1 & 2 & 3 & 4 \\
\hline \multirow{3}{*}{$4 a$} & $\tau_{1}$ & $(1 \overline{1} \overline{1})$ & $(-0.50 .50 .5)$ & $(1 \overline{1} \overline{1})$ & $(1 \overline{1} \overline{1})$ \\
& $\tau_{2}$ & - & - & - \\
& $\tau_{3}$ & $(0.130 .50-0.37)$ & $(0.070 .25-0.19)$ & $(0.130 .50-0.37)$ & $(0.130 .50-0.37)$ \\
\hline \multirow{3}{*}{$4 c$} & $\tau_{1}$ & $(1 \overline{1} \overline{1})$ & $(-0.50 .50 .5)$ & $(1 \overline{1} \overline{1})$ & $(1 \overline{1} \overline{1})$ \\
& $\tau_{2}$ & $-0.50-0.13-0.37)$ & $(-0.25-0.07-0.19)$ & $(-0.50-0.13-0.37)$ & $(-0.50-0.13-0.37)$ \\
& $\tau_{3}$ & $(0.130 .50-0.37)$ & $(0.070 .25-0.19)$ & $(0.130 .50-0.37)$ & $(0.130 .50-0.37)$ \\
& & $(-0.50-0.13-0.37)$ & $(-0.25-0.07-0.19)$ & $(-0.50-0.13-0.37)$ & $(-0.50-0.13-0.37)$
\end{tabular}

формации ГЦК-ГПУ [30]). Присутствие сверхструктурного вклада в признаках двойникования кристаллов на картинах нейтронного рассеяния вполне отвечает формированию сверхструктуры смещения дисторсионного типа, которая, с симметрийной точки зрения, является „промежуточной“ в реконструктивном фазовом переходе. Так, в „близко родственной“ рассматриваемому случаю системе поликристаллов $\mathrm{NiO}-\mathrm{ZnO}$, где первый крайний „чистый“ состав является оксидом $3 d$-металла, а второй формально принадлежит соединениям II-VI, при замещении атомов никеля атомами цинка надежно установлено формирование сверхструктуры [31,32]. Рентген-дифракционные исследования с использованием в том числе и синхротрона выявляют признаки сверхструктуры смещения с волновыми векторами $k=(1 / 31 / 31 / 3) 2 \pi / a$ и $k=(1 / 61 / 61 / 6) 2 \pi / a$.

В общих терминах, принятых для описания фазовых переходов в кристаллических твердых телах, представленные на рис. 2 данные указывают на переходы из ГЦК-решетки по 8-ми лучевой не лифшицевской звезде волнового вектора

$$
k_{5}=\mu\left(b_{1}+b_{2}+b_{3}\right)=(2 \mu \pi / a, 2 \mu \pi / a, 2 \mu \pi / a),
$$

где $b_{1}, b_{2}, b_{3}$ - базисные векторы обратной решетки, а $\mu \approx 1 / 3$ [36].

Расположение дополнительных узлов, относящихся к систематике (1), на схемах рис. 2, $a$ и $c$ выглядит поразному. В таком случае, картины на рис. 2 подразумевают представляемые лучами звезды (2) каналы переходов, различающиеся содержащимися лучами и числом лучей. (При этом каждый из лучей звезды (2) описывает одну и ту же решетку, ориентированную одним из восьми способов относительно исходной решетки.)

Установление звезды волнового вектора из данных дифракционных исследований позволяет указать базисные функции неприводимых представлений (НП) группы симметрии исходной фазы, что важно при рассмотрении фазовых переходов в кристаллах [37,38]. Нами было получено решение этой проблемы для однолучевого канала, на который указывает схема рис. 2, $a$, результаты представлены в табл. 2.
Математический аппарат симметрийного анализа предполагает матрицы НП комплексными, даже если сами представления вещественны. Так, например, использование специальной программы Mody for Windows 1.29 [39] для нахождения базисных функций дает результат в виде проекций на координатные оси, тройкой пар чисел, первое из которых является действительной, а второе - мнимой частью комплексного значения. Однако, для того, чтобы анализировать соответствующие атомные смещения, функции необходимо привести к вещественному виду.

Для операции овеществления не существует сформулированных правил. Поэтому в нашем решении базисные функции в табл. 2 сразу даны вещественными и представляют для каждого атома трехкомпонентный полярный вектор.

Табл. 2 отражает состав векторного представления на позициях $4 a$ и $4 c$ в структуре сфалерита (пространственная группа $F \overline{4} 3 m$ ). Атомы примитивной ячейки имеют следующие координаты.

4a:

1 (0.0 0.0 0.0), 2 (0.0 0.5 0.5), 3 (0.5 0.0 0.5), 4 (0.5 0.5 0.0); $4 c$ :

$1(0.250 .250 .25), 2(0.250 .750 .75), 3 \quad(0.750 .250 .75)$, $4(0.750 .750 .25)$.

Отметим, что значения базисных функций на соответствующих атомах обоих структурных позиций в рассматриваемом случае одинаковы. Рассмотрение переходов, включающих в канал другие лучи звезды (2), предполагает присоединение подобных решений.

Согласно [37] наглядный физический смысл вектора базисной функции - смещение $i$-ого атома (в нашем случае $i=1, \ldots, 4)$ при описании мод нормальных колебаний, в которых атом участвует. Таким образом, данные табл. 2 являются „исходной точкой“ для анализа атомных смещений, создающих предпереходное состояние в кристаллической структуре исследуемых соединений.

Базисные функции одномерного НП $\tau_{1}$ отвечают только радиальным смещениям вдоль луча $k_{2}$. Для результатов нашего эксперимента больший интерес представ- 
ляют функции двумерного НП $\tau_{3}$, отвечающие „поперечным“ атомным смещениям - перпендикулярным направлению $[\overline{1} 11]$, т. е. в плоскости (111) применительно к рассматриваемому случаю. Как видно из данных табл. 2, на каждом атоме структуры пара ортогональных к $\langle\overline{1} 11\rangle$ векторов НП $\tau_{3}$ ортогональна между собой. В общем случае, атомные смещения в плоскостях $\{\overline{1} 11\}$ описываются смешиванием, величины смещений определяются соотношениями компонент пары соответствующих векторов и коэффициентов смешивания.

Необходимость проведенного симметрийного анализа применительно к результатам нашего эксперимента заключается в том, что атомные смещения следует выражать через соответствующие базисные функции. В рамках концепции одного НП, если представление $\tau_{1}$ является ответственным за переход, следует искать координату $x$, которая определяет положения смещенных атомов в позиции 16e [40] добавлением к координатам атомов в позициях $4 a$ и $4 c$ соответствующих базисных функций, умноженных на $x$. При ответственном представлении $\tau_{3}$, в случае атомных смещений в плоскости (111) им будет соответствовать появление точки, общей позиции 96i. Ее координаты $[[x, y, z]]$ следует рассматривать принадлежащими окружности, описываемой относительно начала координат соответствующей атомной компонентой базисной функции, умноженной на коэффициент пропорциональности между величиной истинного смещения и модулем компоненты. Определенные таким образом точки позволят найти коэффициенты смешивания.

Подготовка к моделированию реальной структуры по дифракционным данным табл. 1 на основании вышеизложенного уже дает ненулевую интенсивность дифракционной картины от коррелированных атомных смещений для перечисленных сверхструктурных отражений кристалла $\mathrm{Zn}_{0.95} \mathrm{~V}_{0.05} \mathrm{Se}$ при любых величинах смещений, продольных и поперечных к направлениям $\langle 111\rangle$, и любых соотношениях между количествами смещенных и несмещенных атомов в поврежденных локализованных объемах. Непосредственная характеристика атомных смещений в рассматриваемом случае видится достаточно громоздкой, что заставляет нас отказаться от ее проведения в рамках данной работы. Кроме того, реальные атомные смещения могут удовлетворять предельно общему случаю смешивания между НП $\tau_{1}$ и $\tau_{3}$, что не противоречит общей концепции симметрийного анализа структур при фазовых переходах. Вполне вероятно, предпереходное состояние исследованных кристаллов представимо по отношению к направлениям плотной упаковки послойной укладки и „Поперечными“, и „продольными“ корреляциями в подсистеме смещенных атомов. Именно такая картина наблюдается в системе оксидов $\mathrm{NiO}-\mathrm{ZnO}[31,32]$, когда указанные два типа корреляционной связи проявились разными дифракционными методиками, из-за различия корреляционных длин.

Как видно из рис. $2, a$, результирующая картина расположения узлов на плоскости $\{110\}$ описывается проекцией гексагональной элементарной ячейки (выделена на схеме прямоугольником). При содержании ванадия 0.10, когда расщепленные дополнительные узлы - явный признак тенденции к понижению симметрии, задают две повернутые подрешетки, для описания картины физически правильным видится базис тригональной системы (рис. 2,c, выделенный четырехугольник). В целом, представленные уточненные проекции обратной решетки, предполагающие уменьшение периода в реальном пространстве в 3 раза, указывают на субструктуры.

Полученная нами в настоящей работе нейтронографическая характеристика структуры кристаллов приводит к вопросу, насколько коррелированные атомные смещения, обусловленные дестабилизирующим влиянием со стороны чужеродных ионов в полупроводниковой матрице, связаны с изначальной нестабильностью исходной структуры, ответственной за образование двойников и/или дефектов упаковки? Прямо положив для продольных атомных смещений величину $x=\mu$ (в нашем случае, $\mu \approx 0.33)$, базисные функции НП $\tau_{1}$ переводят $3 / 4$ атомов структуры в центры треугольников, образующих плотноупакованные слои, при этом оставшаяся $1 / 4$ атомов смещается в центры координационных тетраэдров на половину межслоевого расстояния. Таким образом, в рассматриваемой идеализированной структуре бинарных полупроводников, с симметрийной точки зрения, двойникование и/или дефекты упаковки (установление порядка в подсистеме последних ведет, в конечном итоге, к политипии) становятся „подмененными“ физически нереализуемыми положениями атомов.

Согласно данным наших работ [27-29], увеличение плохо растворяющейся $3 d$-примеси в халькогенидах цинка до уровней $\approx 0.05-0.10$ в формульной единице обнаруживает признаки значительных структурных деформаций на макро уровне с величиной, близкой к значениям, при которых исходный материал разрушается. При этом статистика, описывающая структурное состояние кристаллов $\mathrm{Zn}_{1-x} \mathrm{~V}_{x} \mathrm{Se}(x=0.01 ; 0.05 ; 0.10)$, указывает на многочисленные нерегулярные сильные повреждения исходного атомного порядка [29]. В работе [27] при гипотетическом рассмотрении слоевых сдвигов в идеализированной структуре сфалерита на модели „твердых шаров“ мы проиллюстрировали неизбежность появления деформационного поля в сильно легированных $3 d$-примесью кристаллах халькогенидов цинка, привносящей ,электронное“ дестабилизирующее влияние в исходную решетку. Для значительных смещений атомов и/или структурных фрагментов необходимо выполнение двух очевидных условий на качественном уровне: необходимости „свободного пространства“ для локального изменения взаимного расположения атомов и „гибкости“ межатомных связей. Иными словами, необходима „стойкость“ материала к сильным локальным деформациям. Поэтому другой физической основой предпереходного состояния кристаллической структуры обсуждаемых кристаллов следует рассматривать тенденции к изменению электронной структуры, привносимые $3 d$-примесью. Как следует из работ [23,41-44], заметным эффектом 
влияния легирования $3 d$-ионов на исходную решетку кубических кристаллов халькогенидов цинка является „дальнодействие“ электронной деформации, обусловленное чувствительностью к изменениям в распределении электронной плотности вдоль $s p^{3}$-связей за счет гибридизации $d$-состояний примеси с $p$-состояниями аниона. Этот факт, очевидно, должен „смягчать“ ограничивающее условие возможности атомных смещений, налагаемое ковалентностью связей.

В таком случае, суть проблемы изодиморфизма применительно к рассматриваемым соединениям является следующая: локальными атомными смещениями реализуется деформация, отвечающая микронапряжениям, превышающим предел прочности исходного материала. При этом, вновь обращаясь к данным рис. 2 и табл. 1, мы видим, что остается важным вопрос о „пустотах“, необходимых для значительных атомных смещений и/или поворотов атомных кластеров в исходной структуре. В действительности, конечный состав изодиморфического ряда $\mathrm{ZnSe}-\mathrm{VSe}$ является в некоторой степени „открытым“. Общность имеющихся на сегодняшний день фазовых диаграмм систем „ванадий-халькоген“ [45] заключается в изначальной неотъемлемой дефектности получающихся реальных структур, что относит эти соединения к соединениям с равновесной дефектностью или „плавающим“ химическим потенциалом [46]. Общий подход к анализу ансамблей дефектов и их роли в формировании свойств таких систем, включая эффекты упорядочения, содержится в работах $[46,47]$. Так, яркой иллюстрацией проявления присущей реальной структуре монохалькогенидов ванадия дефектности решетки является ослабление магнетизма при постепенном замещении железа ванадием в ряду соединений $\mathrm{Fe}_{7-y} \mathrm{~V}_{y} \mathrm{~S}_{8}$ $(0 \leq y \leq 7)$ [48]. Именно равномерное распределение дефектов катионной подрешетки в структуре типа $\mathrm{NiAs}$, стабилизирующейся в результате легирования ванадием, является одной из главных причин, ведущих к понижению намагниченности.

Набор селенидов ванадия, если опустить проясняемые к сегодняшнему дню детали фазовой диаграммы [45], при нормальных условиях, в общем, представлен тремя фазами: $\alpha$-VSe (структура типа $\mathrm{NiAs}), \beta-\mathrm{V}_{2} \mathrm{Se}_{3}$ (структура с решеткой низшего класса симметрии), $\gamma$-VSe 2 (структура типа $\mathrm{CdI}_{2}$ ), с широкими областями гомогенности при отклонении от характеризующей стехиометрии [49]. В рассматриваемом нами ряду твердых растворов $\mathrm{ZnSe}-\mathrm{VSe}$ будем полагать конечный состав наиболее близким к монохалькогениду, однофазным в пределах от $\mathrm{VSe}_{1.03}$ до $\mathrm{VSe}_{1.25}$ [49], для которого различают идеальную структуру $B 8_{1}$ и „обедненную“ атомами металла $B 31$ [50]. Согласно [50], для селенида ванадия более стабильна нестехиометрическая композиция $\mathrm{V}_{1+x} \mathrm{Se}_{1-y}$ (синтез в условиях, направленных на достижение максимальной фазовой однородности, дает $x \approx 0.12, y \approx 0.16)$, структурный тип которой правильнее назвать ,дефектный NiAs“. При этом решетку, отвечающую $B 81$, стабилизирует избыток атомов металла, заполняющих тригональные бипирамидальные пустоты, и реальная структура предполагает усиление роли связей $\mathrm{V}-\mathrm{V}$ в базовых плоскостях посредством анионных вакансий. Структурное состояние конечного состава изодиморфического ряда $\mathrm{ZnSe}-\mathrm{VSe}$ явно содержит „намек“, что для его „Постройки“ в начальном составе по мере повышения уровня легирования $3 d$-примесью необходима нерегулярная дефектность анионной подрешетки. Следовательно, если по данным электронной микроскопии [51] структурная перестройка „сфалерит-вюрцит“ в чистых халькогенидах цинка протекает под действием спонтанных микродеформаций через активацию систем скольжения $\{111\}\langle 112\rangle$, то в случае легирования кристаллов $3 d$-примесью с сильным дестабилизирующим влиянием (создающей, в общем, направленную деформацию [41-43]) при возникновении предпереходного состояния может оказаться действенным второй механизм фазового перехода ГЦК-ГПУ, через двойникование путем транспозиции [33]. Обсуждение и доказательная критика приемлемости диффузионной модели механизма фазового перехода „сфалерит-вюрцит“ в сульфиде цинка при нагревании представлена работами [51,52]. Именно дополнительная генерация точечных дефектов и дислокаций под сильным деформационным воздействием дестабилизирующей примеси порождающая, в конечном итоге, их двумерные и трехмерные образования в исходной структуре, должна способствовать перемещениям атомов из слоя в слой, при котором „диффузионное просачивание“ будет осуществляться поворотами атомных кластеров и структурных фрагментов.

Отметим, что для более полного понимания механизмов структурной перестройки ГЦК-ГПУ при ее стимулировании чужеродными $3 d$-ионами в кристаллах II-VI и в связи с актуальностью проблемы растворимости магнитоактивной примеси необходимы дальнейшие комплексные исследования.

\section{4. Заключение}

В настоящей работе впервые проведено нейтронографическое исследование при комнатной температуре, характеризующее структурное состояние, предшествующее концентрационному фазовому переходу ГЦК-ГПУ в кристаллах $\mathrm{Zn}_{1-x} \mathrm{~V}_{x} \mathrm{Se}$, с содержанием ванадия $x=0.01,0.05$ и 0.10. Получены данные, указывающие на существование корреляций между атомными смещениями, поперечными к направлению $\langle 111\rangle$, что позволяет считать сверхструктуру с волновыми векторами $k=(1 / 31 / 31 / 3) 2 \pi / a(a-$ параметр кубической ячейки) реально формирующейся. При этом в предпереходное состояние, которое должно реализовываться через промежуточную сверхструктуру дисторсионного типа, вовлечены эффекты, обусловленные изначальной нестабильностью исходной структуры: реальную структуру исследованных сильно легированных кристаллов усложняет множественное двойникование с плоскостями $\{111\}$ и направлениями сдвига $\langle 112\rangle$, один из факторов, определяющих „капризность синтеза“ исходного материала. 
По обнаруженным признакам намечающейся (гипотетической) структурной перестройки, ее возможно отнести к переходу по нелифшицевской звезде, с каналом $k_{5}=\mu\left(b_{1}+b_{2}+b_{3}\right)=(2 \mu \pi / a, 2 \mu \pi / a, 2 \mu \pi / a)$, где $b_{1}, b_{2}, b_{3}$ - базисные векторы обратной решетки, а $\mu \approx 1 / 3$.

\section{Список литературы}

[1] Introduction to the Physics of Diluted Magnetic Semiconductors / Ed. J. Kossut, J.A. Gaj. Springer ser. in Mater. Sci. V. 144. Springer (2010). $469 \mathrm{p}$.

[2] S. Mirov, V. Fedorov, I. Moskalev, D. Martyshkin, C. Kim. Laser Photon. Rev. 4, 21 (2010).

[3] K.S. Lee, G. Oh, E.K. Kim. Solar Energy 164, 262 (2018).

[4] T. Dietl. H. Ohno. Rev. Mod. Phys. 86, 187 (2014).

[5] M. Makkar, R. Viswanatha. Current Scie. 112, 1421 (2017).

[6] P. Kaur, S. Kumar, A. Singh, C.L. Chen, C.L. Dong, T.S. Chan, K.P. Lee, C. Srivastava, S.M. Rao, M.K. Wu. Superlat. Microstruct. 83, 785 (2015).

[7] M. Hassan, S. Younas, F. Sher, S.S. Husain, S. Riaz, S. Naseem. Appl. Phys. A 123, 352 (2017).

[8] D. Saikia, R.D. Raland, J.P. Borah. Physika E: Low-dimens. Syst. Nanostruct. 83, 56 (2016).

[9] J. Yang, F. Muckel, W. Baek, R. Fainblat, H. Chang, G. Bacher, T. Hyeon. J. Am. Chem. Soc. 139, 6761 (2017).

[10] Y.-T. Liu, L.-P. Hou, S.-Y. Zou, L. Zhang, B.-B. Liang, Y.-C. Guo, A. Bukhtiar, M.U. Farooq, B.-S. Zou. Chin. Phys. Lett. 35, 037801 (2018).

[11] М.П. Шаскольская. Кристаллография. Высш. шк., М. (1984). $376 \mathrm{c}$

[12] Полумагнитные полупроводники / Пер. с англ. Под ред. Я. Фурдыны, Я. Косута. Мир, М. (1992). 496 с.

[13] Chin-Yu Yeh, Z.W. Lu, S. Froyen, A. Zunger. Phys. Rev. B 46, 10086 (1992).

[14] В.Ф. Агекян. ФТТ 44, 11, 1921 (2002).

[15] А.С. Пашинкин, Г.Н. Тищенко, И.В. Корнеева, Б.Н. Рыженко. Кристаллография 5, 2, 261 (1960).

[16] Ю.Ю. Логинов, П.Д. Браун, К. Дьюроуз. Закономерности образования структурных дефектов в полупроводниках $\mathrm{A}_{2} \mathrm{~B}_{6}$. Логос, М. (2003). 304 c.

[17] M.T. Sebastian, P. Krishna. Prog. Crystal Growth Charact. Mater. 14, 103 (1987).

[18] В.С. Урусов, Н.Н. Еремин. Кристаллохимия. Краткий курс. Ч. 2. М: Изд-во МГУ, (2005). 125 с.

[19] А. Келли, Г. Гровс. Кристаллография и дефекты в кристаллах / Пер. с англ. Мир, М. (1974). 496 с.

[20] E. Makovicky. Rev. Min. Geochem. 61, 7 (2006).

[21] G. Krishnaiah, N. Madhusudhana Rao, D. Raja Reddy, B.K. Reddy, P. Shreedhara Reddy. J. Cryst. Growth 310, 26 (2008).

[22] T.P. Surkova, S.F. Dubinin, V.I. Maximov, S.A. Lopez-Rivera. Phys. Status Solidi C 9, 8-9, 1830 (2012).

[23] В.И Максимов, С.Ф. Дубинин, В.Д. Пархоменко. Поверхность 2, 9 (2013).

[24] В.И. Максимов, С.Ф. Дубинин, Т.П. Суркова, А.В. Королев. ФТТ 55, 10, 1912 (2013).

[25] В.И. Максимов, С.Ф. Дубинин, Т.П. Суркова. ФТТ 56, 5, 878 (2014).

[26] В.И. Максимов, С.Ф. Дубинин, Т.П. Суркова. ФТТ 56, 12 , 2311 (2014).
[27] В.И. Максимов, Т.П. Суркова, В.Д. Пархоменко, Е.Н. Юшкова. ФТТ 58, 4, 633 (2016).

[28] T. Surkova, V. Maksimov, S. Dubinin, S.A. Lopez-Rivera. Phys. Status Solidi C 13, 456 (2016).

[29] В.И. Максимов, Е.Н. Максимова, Т.П. Суркова. ФТТ 60, 1, 50 (2018).

[30] В.И. Максимов, С.Ф. Дубинин, Т.П. Суркова. Кристаллография 61, 120 (2016).

[31] С.Ф. Дубинин, В.И. Максимов, В.Д. Пархоменко, В.И. Соколов, А.Н. Баранов, П.С. Соколов, Ю.А. Дорофеев. ФТТ 53, 7, 1292 (2011).

[32] В.И. Максимов, С.Ф. Дубинин, А.Н. Баранов, В.И. Соколов, П.С. Соколов, В.Д. Пархоменко. ФММ 114, 799 (2013).

[33] M.E. Fleet. Am. Mineralogist 62, 540 (1977).

[34] E. Michalski, M. Demianiuk, S. Kaczmarek, J. Żmija. Acta Phys. Polonica A 58, 711 (1980).

[35] T. Roisnel, J. Rodriguez-Carvajal. Winplotr, a grafic tool for powder diffraction. LLB, CEA-CNRS, France (2017). http://www.cdifx.univ-rennes1.fr/winplotr/winplotr.htm

[36] О.В. Ковалев. Неприводимые и индуцированные представления и копредставления федоровских групп. Справочное руководство. Наука. М. Гл. ред. физ.-мат. лит., (1986). $368 \mathrm{c}$.

[37] Ю.А. Изюмов, В.Е. Найш, Р.П. Озеров. Нейтроны и твердое тело. Нейтронография магнетиков. Атомиздат, М. (1981). T. 2. $312 \mathrm{c.}$

[38] Ю.А. Изюмов, В.Н. Сыромятников. Фазовые переходы и симметрия кристаллов. Наука, М. (1984). 247 с.

[39] F. Bialas, L. Pytlik, W. Sikora. Open Phys. 14, 559 (2016).

[40] International tables for crystallography. V. A. Space group symmetry / Ed. T. Hahn. The international union of crystallography. Springer (2005). $911 \mathrm{p}$.

[41] С.Ф. Дубинин, В.И. Соколов, С.Г. Теплоухов, В.Д. Пархоменко, Н.Б. Груздев. ФТТ 48, 2151 (2006).

[42] С.Ф. Дубинин, В.И. Соколов, С.Г. Теплоухов, В.Д. Пархоменко, В.В. Гудков, А.Т. Лончаков, И.В. Жевстовских, Н.Б. Груздев. ФТТ 49, 1177 (2007).

[43] V. Gudkov, A. Lonchakov, V. Sokolov, I. Zhevstovskikh. J. Korean Phys. Soc. 53, 63 (2008).

[44] V.V. Gudkov, I.B. Bersuker. In: M. Atanasov et al. (eds). Vibronic Interactions and the Jahn-Teller Effect: Theory and Applications. Progress in Theoretical Chemistry and Physics 23, 143. Springer Science + Business Media B.V. 2012.

[45] J.F. Smith. In: Binary Alloy Phase Diagrams (on CD). Second edition plus updates (Based on 1990 threevolume set Binary Alloy Phase Diagrams, Second edition, T.B. Massalski, H. Okamoto, P.R. Subramanian, L. Kacprzak eds.). ASM/NIST Data Program for Alloy Phase Diagrams. ASM International, USA, 1996.

[46] Ф. Крегер. Химия несовершенных кристаллов. Мир, М. (1969). $656 \mathrm{c}$.

[47] А.А. Ремпель, А.И. Гусев. Нестехиометрия в твердом теле. Физматлит, М. (2018). 640 с.

[48] N.V. Selezneva, P.N.G. Ibrahim, N.M. Toporova, E.M. Sherokalova, N.V. Baranov. Acta Physica Polonica A 133, 450 (2018).

[49] Д.М. Чижиков, В.П. Счастливый. Селен и селениды. Наука, М. (1964).

[50] L.H. Lewis, J.B. Goodenough. J. Solid State Chem. 114, 346 (1995).

[51] M. Akizuki. Am. Mineral. 66, 1006 (1981).

[52] M. Akizuki. Am. Mineral. 68, 847 (1983).

Редактор Ю.Э. Китаев 